\title{
An objective method for selecting command sources for myoelectrically triggered lower-limb neuroprostheses
}

\author{
Anirban Dutta, PhD; ${ }^{1-2 *}$ Rudi Kobetic, MS; ${ }^{1}$ Ronald J. Triolo, $\mathbf{P h D}^{1-2}$ \\ ${ }^{1}$ Louis Stokes Cleveland Department of Veterans Affairs Medical Center, Motion Study Laboratory, Cleveland, OH; \\ ${ }^{2}$ Departments of Orthopaedics and Biomedical Engineering, Case Western Reserve University, Cleveland, $\mathrm{OH}$
}

\begin{abstract}
Functional electrical stimulation (FES) facilitates ambulatory function after paralysis of persons with spinal cord injury (SCI) by exciting the peripheral motor nerves to activate the muscles of the lower limbs. This study identified a process for selecting command sources for triggering FES with the surface electromyogram (EMG) from muscles partially paralyzed by incomplete SCI, given its high degree of intersubject variability. We found Discriminability Index (DI) to be a good metric to evaluate the potential of controlling FES-assisted ambulation in four nondisabled volunteers and two participants with incomplete paralysis. The left erector spinae $(E S)$ (mean DI $=0.87$ ) for triggering the left step and the right ES (mean DI $=0.83$ ) for triggering the right step were the best command sources for participant 1 . The left ES (mean DI $=0.93$ ) for triggering the left step and the right medial gastrocnemius (mean DI $=0.88$ ) for triggering the right step were the best command sources for participant 2 . Our results showed that command sources can be selected objectively from surface EMG before a fully implantable EMG-triggered FES system for walking is implemented.
\end{abstract}

Key words: Discriminability Index, electromyogram, functional electrical stimulation, gait, implantable stimulator-telemeters, incomplete spinal cord injury, motor relearning, myoelectric control, pattern recognition classifier, rehabilitation.

\section{INTRODUCTION}

Functional electrical stimulation (FES) provides wheelchair-dependent individuals an opportunity for brace-free ambulation with incomplete spinal cord injuries (iSCIs). Neuroprostheses using FES can electrically activate a customized set of muscles selected by a clinician to address individual gait deficits with preprogrammed patterns of stimulation to produce cyclic movement of the lower limbs for ambulation [1-2]. Users normally use a switch to trigger each step manually and progress through the customized pattern of muscle activations required to achieve walking function. In this study, we evaluated the ability of both nondisabled volunteers and individuals with iSCI to determine the intent to step during level overground walking using surface electromyogram (EMG). Our goal was to specify a process and criterion for selecting two muscles for a new command and control interface that can be implemented with only two channels of implanted EMG recording electrodes to coincide with the technical capabilities of

\footnotetext{
Abbreviations: ANOVA $=$ analysis of variance, ASIA $=$ American Spinal Injury Association, BF = biceps femoris, $\mathrm{C}=$ cervical, DI = Discriminability Index, EMG = electromyogram, ES = erector spinae, FES = functional electrical stimulation, $\mathrm{GM}=$ gluteus medius, IRS = Implantable Receiver Stimulator, iSCI = incomplete spinal cord injury, LE = linear envelope, $\mathrm{MG}=$ medial gastrocnemius, $\mathrm{PRC}=$ pattern recognition classifier, $\mathrm{RF}=$ rectus femoris, $\mathrm{ROC}=$ Receiver Operating Characteristic, $\mathrm{SD}=$ standard deviation, $\mathrm{T}=$ thoracic, $\mathrm{TA}=$ tibialis anterior, $\mathrm{TC}=$ threshold-based classifier.

* Address all correspondence to Anirban Dutta, PhD; Rehabilitation Institute of Chicago, Neural Engineering Center for Artificial Limbs, 345 E Superior St, Chicago, IL 60611; 571-299-0626; fax: 312-238-2081. Email: adutta@ieee.org DOI:10.1682/JRRD.2010.08.0141
} 
currently available implantable technology [3-6]. This article summarizes the method for evaluating the surface EMG from partially paralyzed muscles of two volunteers with iSCI and its comparison with normative data from four nondisabled participants.

While detecting gait event is possible with physical sensors such as force sensitive resistors, accelerometers, and gyroscopes [7-8], biopotentials such as EMG can also provide useful and reliable information when the movement is impaired [9-11]. The EMG temporally precedes the generation of force in a muscle and the resulting movement of a joint [12]. This precedence makes EMG an attractive signal for detecting intent and can allow FES to assist the desired movement. Graupe and Kordylewski presented a neural network-based classifier with online learning capabilities for individuals with complete paraplegia that used EMG signals from nonparalyzed muscles above the level of the spinal cord lesion (primarily the trunk and shoulder muscles) as the command sources [11,13]. Thorsen et al. showed that wrist extension improved by controlling FES with surface EMG from partially paralyzed wrist extensors [14]. Futami et al. showed the feasibility of proportional control of FES with the surface EMG from the same muscle (partially paralyzed knee extensors) in incomplete hemiplegia [15]. Our research group conducted a preliminary study that demonstrated the feasibility of FES-assisted walking triggered by the surface EMG from partially paralyzed muscles [5]. However, given both the high degree of variability observed in the iSCI population and the limited number of recording channels available in implantable neuroprostheses, an objective and quantitative method for comparing and selecting the command sources for an implanted gait-assist system is required. A metric taken from signal detection theory called the Discriminability Index (DI) is presented in this article. We used DI to rank the partially paralyzed muscles according to their ability to detect the intent to step during level overground walking.

\section{METHODS}

\section{Participants}

Two male participants with iSCI volunteered for this study. Participant iSCI-1 was a 23-year-old male with C7 (seventh cervical $[\mathrm{C}]$ vertebra) motor and C6 sensory iSCI (American Spinal Injury Association [ASIA] C), resulting in bilateral paralysis, who could stand but could not initiate a step without help from FES. Participant iSCI-2 was a 34-year-old male with T1 (first thoracic [T] vertebra) motor and C6 sensory iSCI (ASIA D), resulting primarily with left-side paralysis and right-side weakness. Participant iSCI-2 could walk only short distances without the help from FES. Each participant received an eight-channel Implantable Receiver Stimulator (IRS) (IRS-8) and eight surgically implanted intramuscular electrodes in a related study designed to facilitate household and limited community ambulation [16-17]. Four male nondisabled participants with a mean age of 39.5 years (ranging from 25 to 54 years) provided the normative data for comparison. The nondisabled participants had no known injury or pathology to either lower limb before or during the study.

Participant iSCI-1 received an IRS and eight intramuscular stimulating electrodes (IRSs) bilaterally to recruit iliopsoas, vastus intermedius and lateralis, tensor fasciae latae, tibialis anterior (TA), and peroneus longus muscles. Participant iSCI-2 received an IRS with eight stimulating electrodes only on his left side to recruit iliopsoas, vastus intermedius and lateralis, tensor fasciae latae, gluteus medius (GM), gluteus maximus, posterior portion of adductor magnus, and TA (two electrodes). We customized temporal patterns of stimulation to activate the muscles for their particular gait deficits according to established tuning procedures to achieve forward stepping in a rolling walker [18-19]. The participants completed 6 weeks of overground gait training ( $2 \mathrm{~h}$ sessions, 3 times a week) using the implanted FES system with the help of a physical therapist. Details of the standardized course of overground gait training with FES have been summarized in previous publications [16-17]. After discharge from rehabilitation, the participants volunteered for additional studies of the potential for controlling the implanted neuroprostheses with the myoelectric signals recorded from their partially paralyzed lower-limb musculature.

\section{EMG Signal Acquisition and Processing}

The experimental setup is shown in Figure 1. Participants with iSCI walked with an implanted switchtriggered FES system based on the IRS-8 implanted pulse generator and controlled by an external control unit while the activity of the partially paralyzed musculature was monitored by way of surface recording electrodes.

Surface EMG signals were collected from GM, biceps femoris (BF), medial gastrocnemius (MG), rectus femoris (RF), TA, and erector spinae (ES) (at T9) bilaterally. 


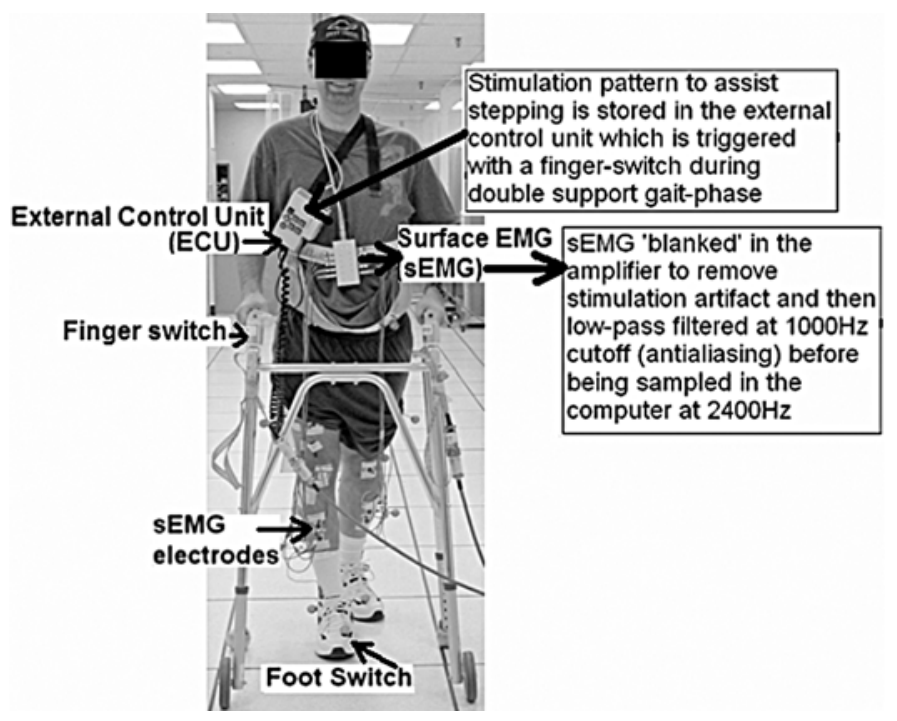

Figure 1.

Experimental setup for surface electromyogram (sEMG) data collection with switch-triggered functional electrical stimulationassisted overground walking.

In case of participants with iSCI, the surface EMG was collected during switch-triggered FES-assisted gait when each step was initiated by a depression of ring-mounted finger switch. Nondisabled volunteers were instructed to walk at a comfortable self-selected speed while the EMG signals of the same muscles were monitored on their right side only.

We collected surface EMG using Ag/AgCl (silver metal and silver chloride) electrodes with $2 \mathrm{~cm}$ interelectrode distance following the SENIAM (Surface ElectroMyoGraphy for the Non-Invasive Assessment of Muscles) [20]. The EMG signals were amplified and low-pass filtered (antialiasing, frequency cutoff $=1,000 \mathrm{~Hz}$ ) by CED 1902 amplifiers (Cambridge Electronic Design; Cambridge, England) before being sampled at 2,400 Hz. The CED 1902 amplifier has a switching circuit (clamp) that was activated by a trigger pulse that disconnected the electrode inputs from the amplifier and connected them to the common electrode just before the start of the stimulation pulse. We clamped the input channels of CED 1902 this way when stimulation pulses were applied to the muscles to prevent stimulation artifact. We set the gain of each channel separately in the CED 1902 amplifiers to prevent saturation at the maximum muscle activity during the gait cycle.

During each trial, the participants were asked to start walking after standing for $3 \mathrm{~s}$ and reach a self-selected steady state speed within $5 \mathrm{~m}$ from the start position.
After reaching the self-selected steady state speed, the participants had to decelerate and return to standing. The participants were then asked to wait in terminal stance for 2 to $3 \mathrm{~s}$. In case of participants with iSCI, the goal was to collect about 10 trials for each session (a day of experiments), and three such sessions were evenly spread over a week. The gait trials were interspaced with adequate rest periods, and the number of trials during a session was based on verbal feedback from each participant with iSCI. The participants with iSCI were motivated to use their partially paralyzed muscles as much as they could during FES-assisted walker-aided ambulation. Data were collected over a month to capture day-to-day variability, which resulted in 60 trials for each participant with iSCI. We collected 15 trials for each session (a day of experiments) for each participant from the four nondisabled participants, resulting in 60 total trials.

The processing of the data was performed offline, which is illustrated in Figure 2 and explained in the following. The implanted FES system delivered electrical pulses at a frequency of $20 \mathrm{~Hz}$, so the sampled EMG was divided into bins of $50 \mathrm{~ms}$ duration. In each bin, we blanked $30 \mathrm{~ms}$ following the start of the stimulation pulse to remove the residual stimulation artifact and $\mathrm{M}$ wave, thus leaving the signal related to the voluntary muscle activity (Figure 2(a)). The blanked portion of the EMG was reconstructed (Figure 2(b)) with the average value of the EMG in the preceding and succeeding blocks [21]. We then low-pass filtered the whole EMG pattern (5th order zero-lag Butterworth, frequency cutoff $=3 \mathrm{~Hz}$ ) to extract the linear envelope (LE) (Figure 2(c)). The EMG pattern for each muscle was normalized by the maximum value of the EMG LE during gait cycle. The normalized LEs during a gait cycle were then divided into doublesupport and swing phase of gait based on the occurrence of foot strike and foot off. The foot and ground contact sequences were determined from insole foot switches (B\&L Engineering; Santa Ana, California) placed bilaterally at the medial and lateral heel, first and fifth metatarsal, and big toe. One can detect the intent to step based on the magnitude of the LE when it crosses a selected threshold (i.e., threshold-based classifier [TC]) or by matching the LE pattern with a specified (characteristic) pattern of muscle activity using cross-correlation analysis (i.e., pattern recognition classifier [PRC]). The LE pattern for participant iSCI-1 has been presented in Dutta et al. [5], and the LE pattern for participant iSCI-2 is shown in Figure 3. The ballistic stepping with the lower limbs 

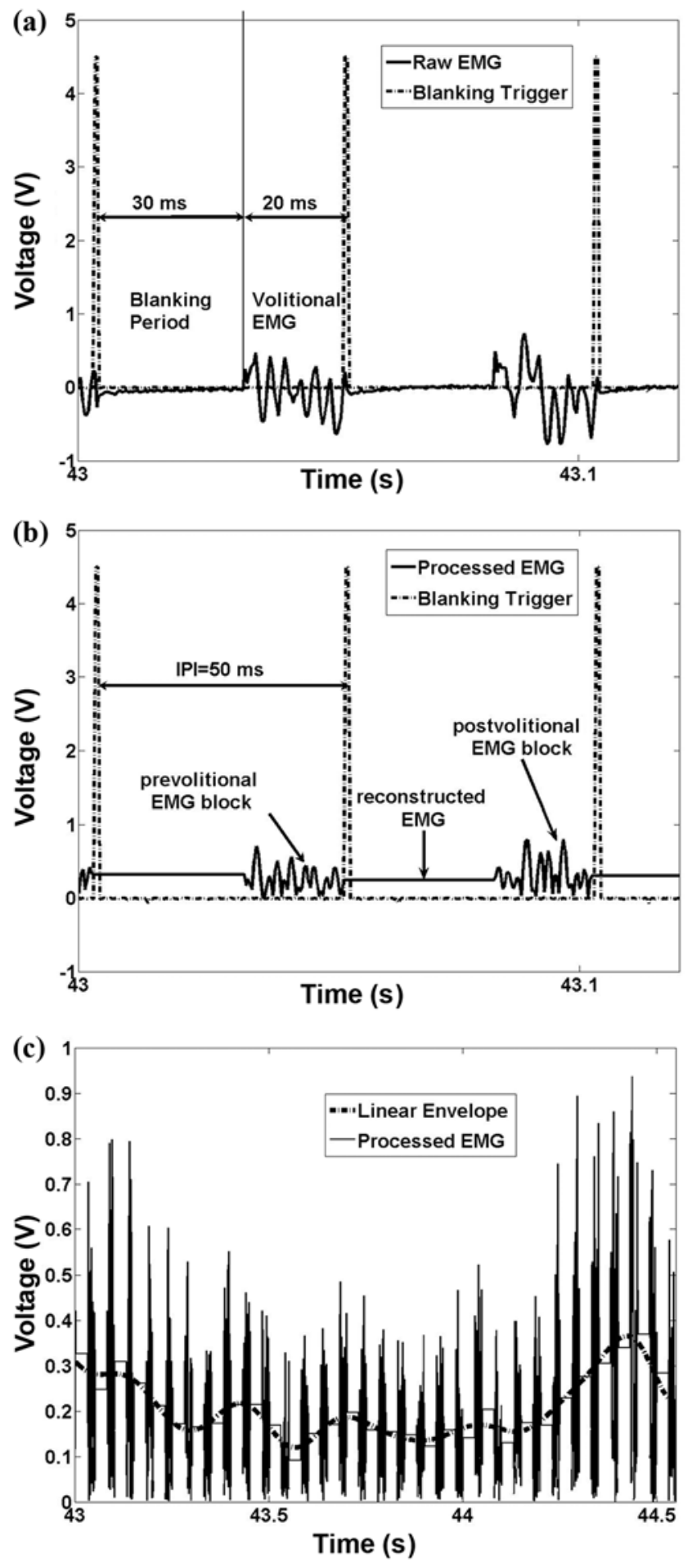

Figure 2.

Processing of raw electromyogram (EMG) collected during functional electrical stimulation to find its linear envelope (LE): (a) Raw EMG with blanking trigger, (b) processed EMG with blanked portions reconstructed with average EMG from pre- and postvolitional blocks, and (c) LE found from processed EMG. IPI = interpulse interval.
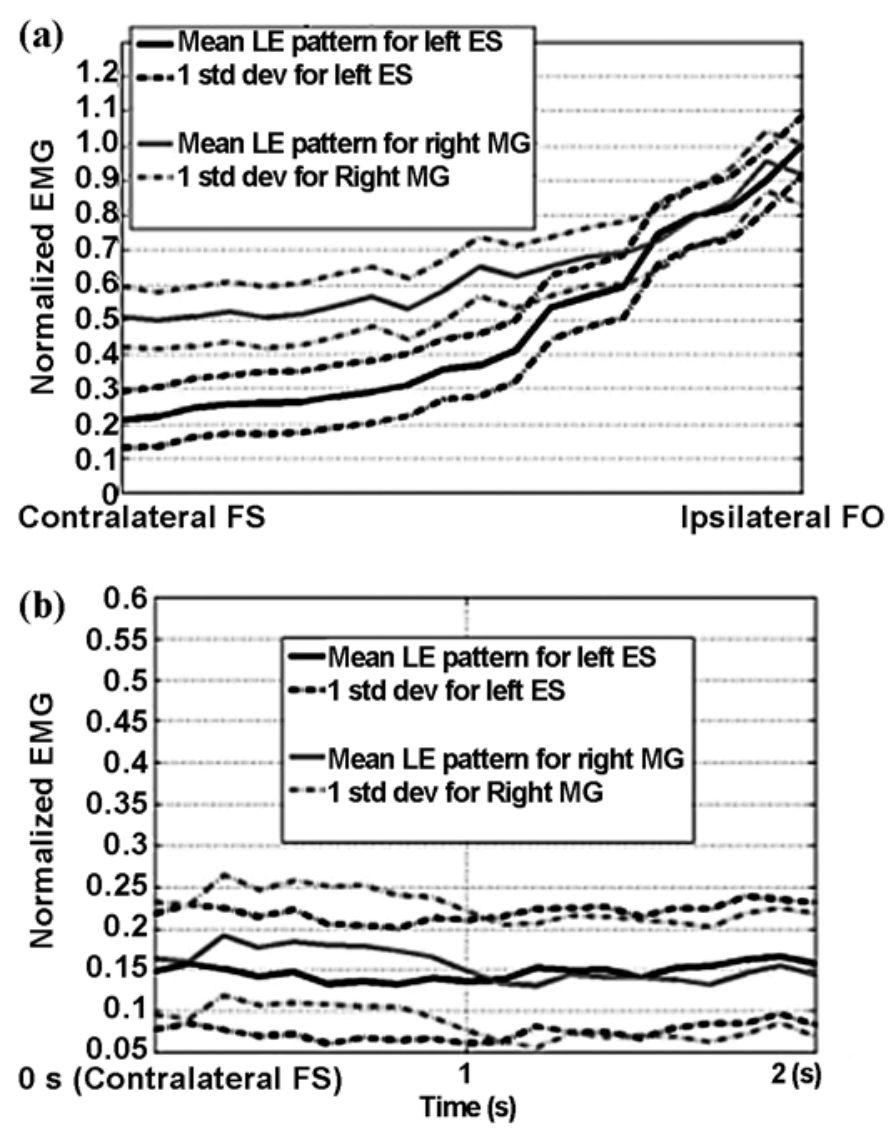

Figure 3.

Characteristic linear envelope (LE) pattern for triggering functional electrical stimulation for participant 1 with incomplete spinal cord injury: LE characteristic patterns for (a) class "true" $(N=150)$ and (b) "false" $(N=150)$. EMG = electromyogram, ES = erector spinae, $\mathrm{FO}$ = foot off, $\mathrm{FS}$ = functional stimulation, $\mathrm{MG}$ = medial gastrocnemius, std dev = standard deviation.

was assisted with preprogrammed temporal patterns of FES, which were triggered with EMG.

\section{Selection of Command Sources}

The normalized LEs of the EMG from each muscle were divided into two classes: the class "True" was composed of LEs during the double-support phase before foot off and the class "False" consisted of the LEs during all other activities, including terminal stance, swing, and quietstanding. We randomly allocated half the data to training and used it to find a characteristic pattern of activation by ensemble-averaging the LEs. The characteristic pattern found for the class True was cross-correlated with the LEs from the other half of the data (test data) for the classesTrue and False. We computed Receiver Operating 
Characteristic (ROC) curve for each of the candidate muscles to assess the trade-off between the True Positive Rate (sensitivity) and False Positive Rate ( $1=$ specificity) of both TCs and pattern recognition-based binary classifiers, while the decision threshold was varied [22-24]. For the PRC, the ROC curve was computed from the crosscorrelation coefficient of the characteristic pattern with the LEs for the True and False classes, whereas for the TC, the ROC curve was computed from the amplitude of the LEs for the True and False classes.

The LEs from the surface EMG signals of the left and right sides of all four nondisabled participants were considered symmetrical. Therefore, the performances of the PRC and TC were evaluated only for the right side in the case of nondisabled participants.

The area under the ROC curve exhibits a number of desirable properties for characterizing overall accuracy of binary classifiers - it is independent of the decision threshold and invariant to a priori class probabilities [25]. The area under the ROC curve, also called the DI, was numerically computed with trapezoidal integration. For cases where $0.5 \mathrm{DI}<1$, the mean of the True class data was greater than or equal to the mean of the False data and the values greater than the discrimination threshold were classified as True. Therefore, DI indicated how well a simple binary classifier could discriminate between the intent to step (True) and the intent to stand (False) during the double-support phase of gait.

DIs for the PRC (DI $\left.{ }_{\mathrm{PRC}}\right)$ and $\mathrm{TC}\left(\mathrm{DI}_{\mathrm{TC}}\right)$ were derived from the areas under the ROC curves generated for each EMG signal source. The data were randomly partitioned 10 times into training and test data sets for a 10 -fold cross validation. For consistency, the same training and test data sets were used by both PRCs and TCs, for computing the ROC curves in a paired experimental design. Therefore, we generated 10 ROC curves for each classifier by randomly pooling the LEs into training and test data sets. The DI was computed for each ROC curve and then averaged to find the mean $\left(\mathrm{DI}_{\mathrm{PRC}}\right.$ and $\left.\mathrm{DI}_{\mathrm{TC}}\right) \pm$ standard deviation (SD) ( $\mathrm{SD}\left(\mathrm{DI}_{\mathrm{PRC}}\right)$ and $\mathrm{SD}\left(\mathrm{DI}_{\mathrm{TC}}\right)$ ) for each classifier.

\section{Statistical Analysis}

We performed a two-way two-tailed analysis of variance (ANOVA) (ANOVA2 in MATLAB R14 [The MathWorks, Inc; Natick, Massachusetts]) on the DIs computed from the walking data. All observations were considered mutually independent for the ANOVA test. The $p$-value was computed for the null hypotheses:

- $\mathrm{H}_{0}$ : The DIs for the PRC and TC have equal mean values.

- $\mathrm{H}_{2}$ : The DIs for all the muscles have equal mean values.

- $\mathrm{H}_{0}$ : No interactions are between the classifier type and muscles selected.

If the $p$-value was close to zero $(<0.05)$, then that null hypothesis was rejected and the result was considered statistically significant. To find which pairs were significantly different, we performed post hoc tests. The critical values for simultaneous comparison of all linear combinations of mean values were found from the conservative Scheffé $S$ procedure with a significance level of $0.05(\alpha=0.05)$.

\section{RESULTS}

Table 1 depicts the mean \pm SD values of the DIs for nondisabled participants by muscle and classifier type. The value of DI was equal to zero for TC when the amplitude

Table 1.

Mean \pm SD values of DI for nondisabled participants $(n=4)$ by muscle and classifier type. Muscles tested were GM, BF, MG, RF, TA, and ES (at 9th thoracic vertebra) and classifiers were PRC and TC. SD of DI was over 10 random partitions (i.e., 10 -fold cross validation).

\begin{tabular}{lcc}
\hline $\begin{array}{c}\text { Muscles for Right-Step } \\
\text { Classifier for } \\
\text { Nondisabled }\end{array}$ & $\begin{array}{c}\mathbf{D I}_{\mathbf{P R C}} \pm \mathbf{S D} \\
\text { (DI }\end{array}$ & $\begin{array}{c}\mathbf{D I}_{\mathbf{P R C}} \mathbf{\text { TC }} \\
\mathbf{( D I}_{\mathbf{T C}} \mathbf{S D}\end{array}$ \\
\hline Participants & $0.42 \pm 0.00$ & $0.00 \pm 0.00$ \\
Ipsilateral GM & $0.29 \pm 0.00$ & $0.00 \pm 0.00$ \\
Ipsilateral BF & $1.00 \pm 0.00$ & $1.00 \pm 0.00$ \\
Ipsilateral MG & $0.74 \pm 0.01$ & $0.59 \pm 0.05$ \\
Ipsilateral TA & $0.52 \pm 0.00$ & $0.00 \pm 0.00$ \\
Ipsilateral ES & $1.00 \pm 0.00$ & $1.00 \pm 0.00$ \\
Contralateral GM & $1.00 \pm 0.00$ & $1.00 \pm 0.00$ \\
Contralateral BF & $1.00 \pm 0.00$ & $1.00 \pm 0.00$ \\
Contralateral MG & $0.26 \pm 0.00$ & $0.00 \pm 0.00$ \\
Contralateral RF & $1.00 \pm 0.00$ & $0.42 \pm 0.04$ \\
Contralateral TA & $1.00 \pm 0.00$ & $1.00 \pm 0.00$ \\
Contralateral ES & $0.49 \pm 0.02$ & $0.00 \pm 0.00$
\end{tabular}

$\mathrm{BF}=$ biceps femoris, $\mathrm{DI}=$ Discriminability Index, $\mathrm{ES}=$ erector spinae, $\mathrm{GM}=$ gluteus medius, $\mathrm{MG}=$ medial gastrocnemius, $\mathrm{PRC}=$ pattern recognition classifier, $\mathrm{RF}=$ rectus femoris, $\mathrm{SD}=$ standard deviation, $\mathrm{TA}=$ tibialis anterior, $\mathrm{TC}=$ threshold-based classifier. 
of LE in the True class was lower than that in the False class. This result is because we were interested in those muscles that the participant activates to trigger the stepping action, and therefore, the mean of the True class data has to be greater than or equal to the mean of the False data. We found statistically significant differences in the mean values of DI because of muscle as well as the classifier type. Results from the post hoc analysis are presented in Figure 4. Ipsilateral MG and ES and contralateral BF, GM, and TA all performed equally well (mean DI $=1$ ) as command inputs (Figure 4(a)). Figure 4(b) shows that the PRC (mean $\mathrm{DI}_{\mathrm{PRC}}=0.76$ ) performed much better than the TC (mean $\mathrm{DI}_{\mathrm{TC}}=0.5$ ) during walking of nondisabled participants.

The DI of some of the muscles of both the participants with iSCI improved during the month-long data collection. Twelve 1-day sessions were spread over a month. We used half the trials during each session to train the classifiers and the other half to compute the DI. Figure 5 shows the changes in the DI over the period of data collection for the participant iSCI-1, whereas Figure 6 shows the time course of variations in DI for the participant iSCI-2. Figures 5(a)-(b) and 6(a)-(b) show the results from the left-step classifier, and Figures 5(c)-(d) and 6(c)-(d) show the results from the right-step classifier. Figures 5(a) and (c) and 6(a) and (c) show the results from the TC, whereas Figures 5(b) and (d) and 6(b) and (d) show the results from the PRC. We noted that the DI improved for the ipsilateral MG and ipsilateral ES muscles of the participant iSCI-1, regardless of the classifier applied (i.e., both muscles performed equally well in either the TC or PRC). The left ES for the left-step TC and PRC and the right ES for the right-step TC and PRC had the highest DI during most sessions for participant iSCI-1.

The DI improved similarly for both TC and PRC for participant iSCI-2, as well (Figure 6). As shown in Figure 6, the leading muscles for PRC command sourceipsilateral MG, ipsilateral ES, and contralateral RF (ipsilateral and contralateral are defined with respect to the classifier; for example, for left-step classifier, left MG, left ES, and right RF were the best)—had comparable DI during most sessions. Also, the left-step PRC that was the more involved side improved more noticeably than the right-step PRC. The DI of the leading muscles for PRC took about eight sessions to reach steady state (DI > 0.95) for the bilaterally involved participant iSCI-1, whereas

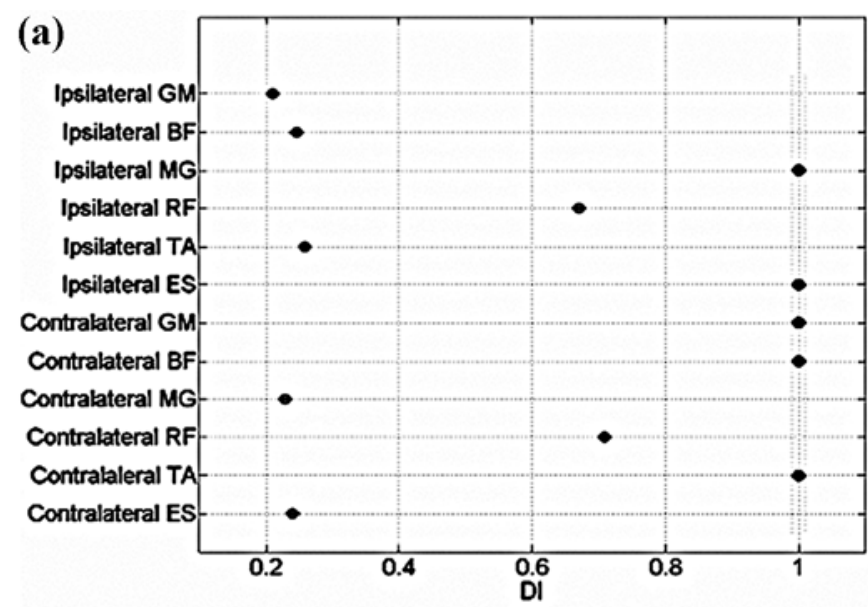

(b)

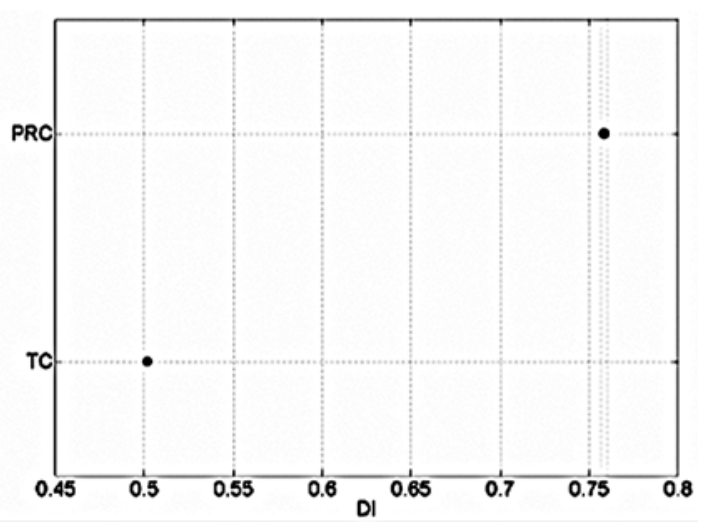

Figure 4.

Post hoc analysis of Discriminability Index (DI) with critical values $(\alpha=$ 0.05 ) from Scheffé S procedure by (a) muscle for right-step classifier and (b) classifier type for right-step trigger for nondisabled participants. Muscles tested are gluteus medius (GM), biceps femoris (BF), medial gastrocnemius (MG), rectus femoris (RF), tibialis anterior (TA), and erector spinae (ES) (at 9th thoracic vertebra). Binary classifiers tested are pattern recognition classifier (PRC) and threshold-based classifier (TC). True mean with 95\% confidence interval is shown for highlighted (a) muscle and (b) classifier and others in pairwise comparison. Mean values are significantly different if their intervals do not overlap.

the DI took about four sessions for the unilaterally involved participant iSCI-2.

Data from all trials during the month-long data collection were pooled together for a 10 -fold cross validation. Table 2 lists the mean \pm SD values of the DI of the left- and right-step classifiers for participant iSCI-1 by muscle and classifier type. The performance of the TC and PRC for this participant with incomplete paralysis generally paralleled those for nondisabled participants. We found statistically significant differences in the mean values of DI because of muscle as well as the classifier type. The results from the 
(a)

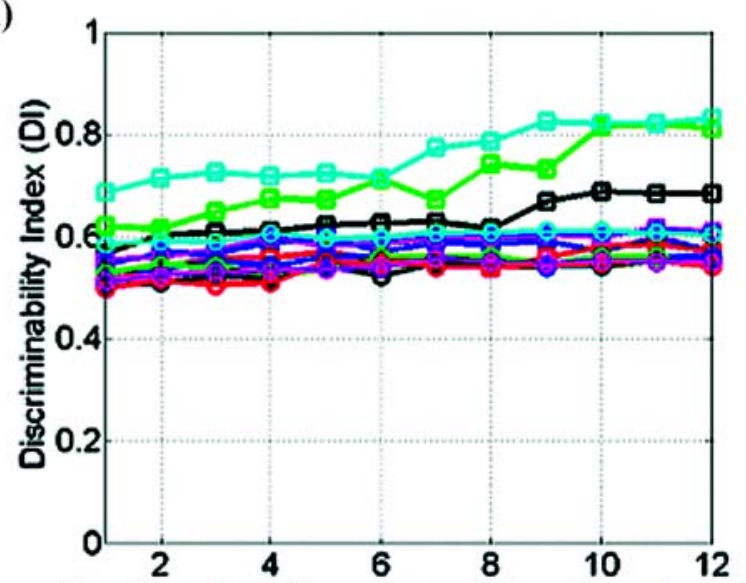

No. of sessions ( 1 session $=1$ day of experiment)

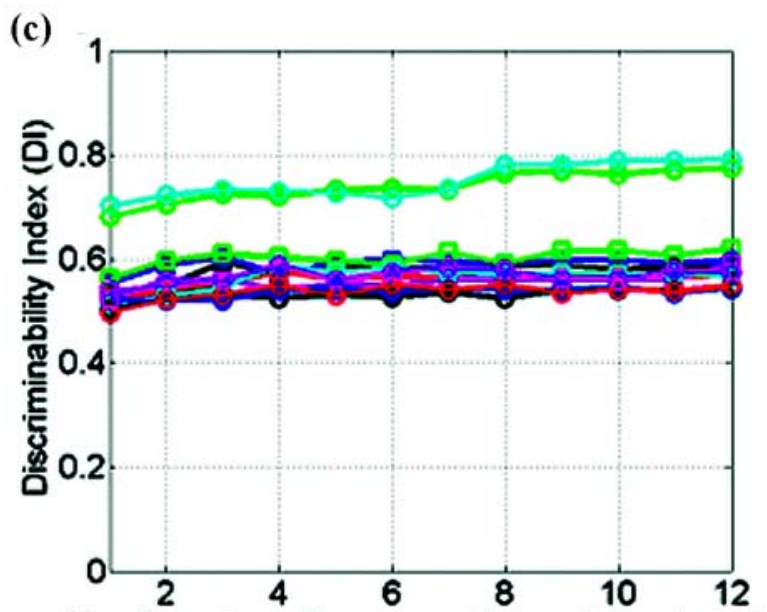

No. of sessions ( 1 session $=1$ day of experiment) (b)

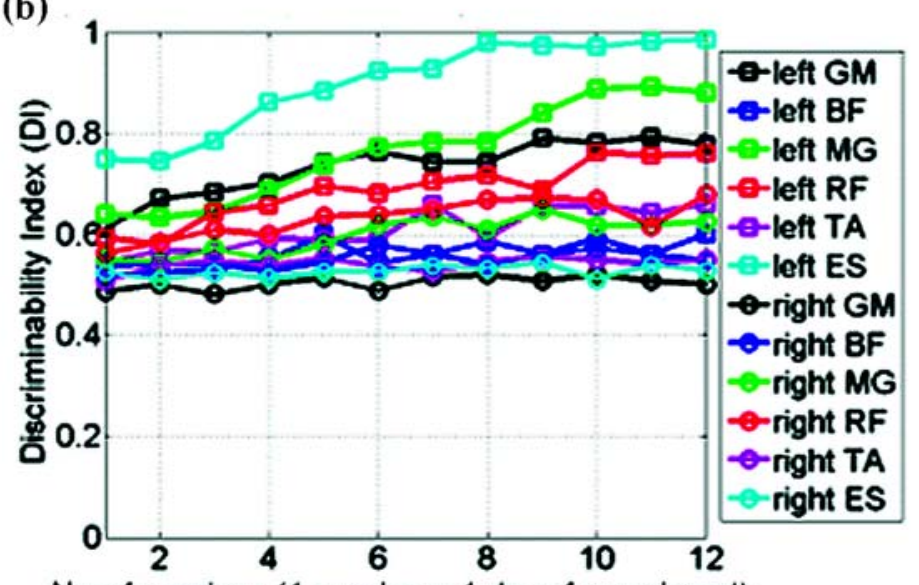

No. of sessions ( 1 session $=1$ day of experiment)

(d)

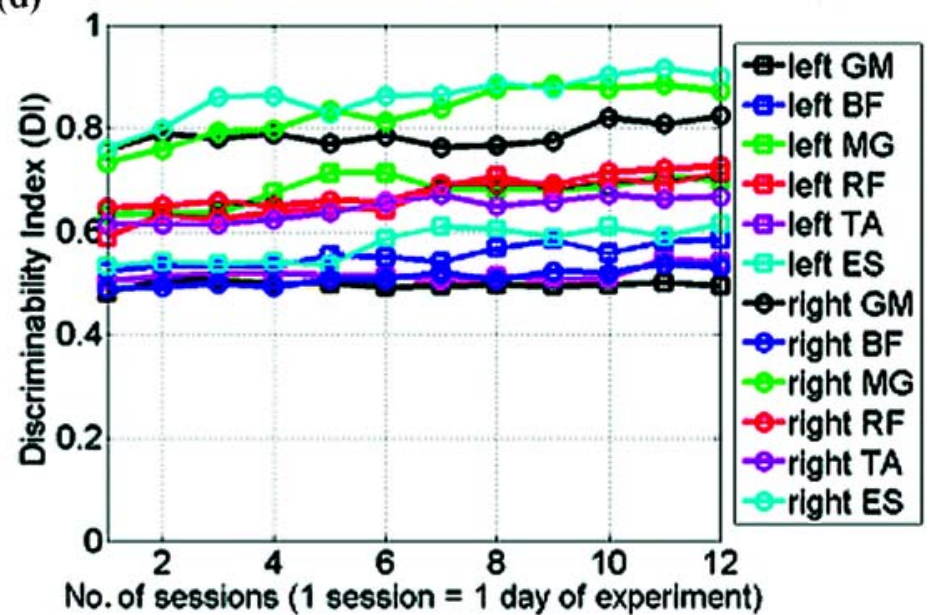

Figure 5.

Session-to-session changes in DI during month-long data collection for participant 1 with incomplete spinal cord injury (total 12 sessions): (a) left-step threshold-based classifier (TC), (b) left-step pattern recognition classifier (PRC), (c) right-step TC, and (d) right-step PRC. (For leftstep classifiers, left side is ipsilateral and right side is contralateral. For right-step classifiers, right side is ipsilateral and left side is contralateral.) $\mathrm{BF}=$ biceps femoris, $\mathrm{ES}=$ erector spinae, $\mathrm{GM}=$ gluteus medius, $\mathrm{MG}=$ medial gastrocnemius, $\mathrm{RF}=$ rectus femoris, $\mathrm{SD}=$ standard deviation, $\mathrm{TA}=$ tibialis anterior.

post hoc analysis are presented in Figure 7. The ES and MG were the preferred command inputs, regardless of the side for this bilaterally involved participant, and the PRC slightly outperformed the TC. The left ES (mean DI $=0.87$ ) performed the best followed by the left MG (mean DI = 0.79) for command source inputs for the left step, and the PRC (mean $\mathrm{DI}_{\mathrm{PRC}}=0.65$ ) performed slightly better than the TC (mean $\mathrm{DI}_{\mathrm{TC}}=0.60$ ), as indicated in Figure 7(a) and (c). For the right step, right ES (mean DI $=0.83$ ) performed the best, followed by the right MG (mean DI $=0.80$ ) as command inputs, and the PRC (mean $\mathrm{DI}_{\mathrm{PRC}}=0.66$ ) per- formed slightly better than the $\mathrm{TC}$ (mean $\mathrm{DI}_{\mathrm{TC}}=0.59$ ), as shown in Figure 7(b) and (d).

Table 3 lists the mean \pm SD values of the DI of the left- and right-step classifiers for iSCI-2 by muscle and classifier type. These outcomes were consistent with the results obtained for both the nondisabled group and participant iSCI-1. We found statistically significant differences in the mean values of DI because of both muscle and classifier type. The results from the post hoc analysis are presented in Figure 8. The left ES (mean DI =0.93) was the best command source for the left step, followed 
(a)

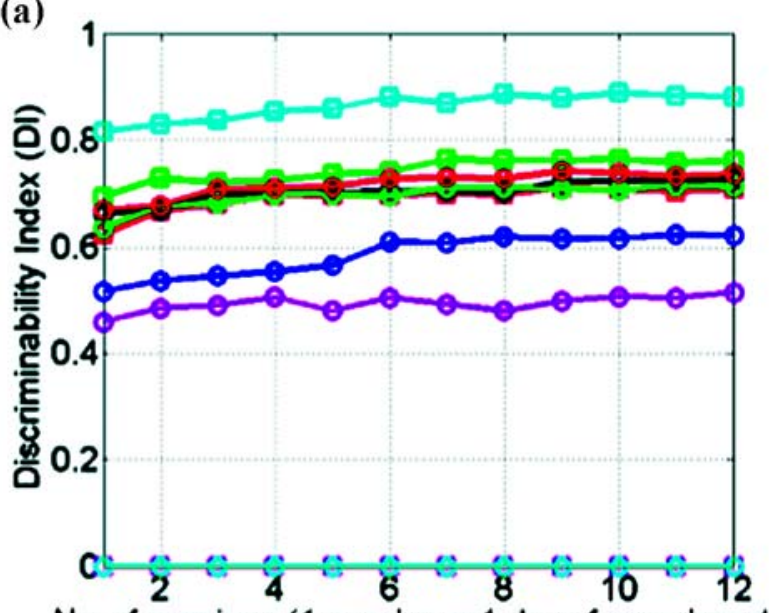

No. of sessions ( 1 session $=1$ day of experiment)

(c)

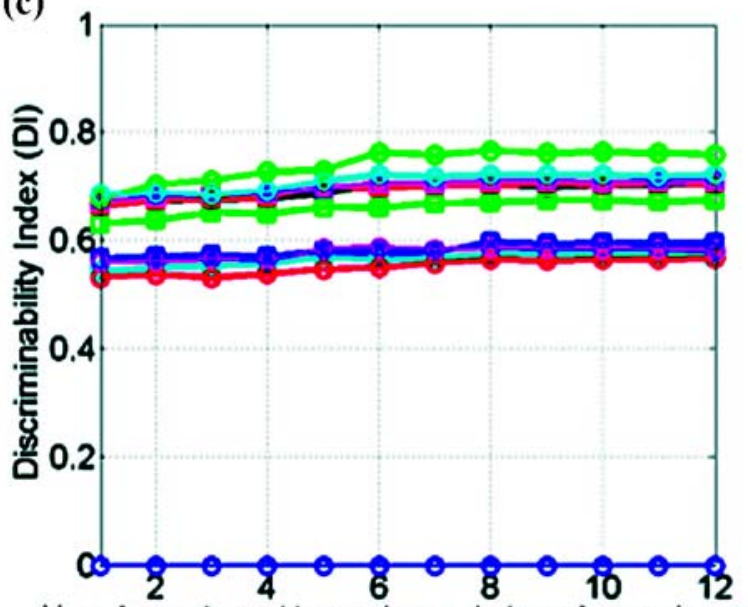

No. of sessions ( 1 session $=1$ day of experiment) (b)

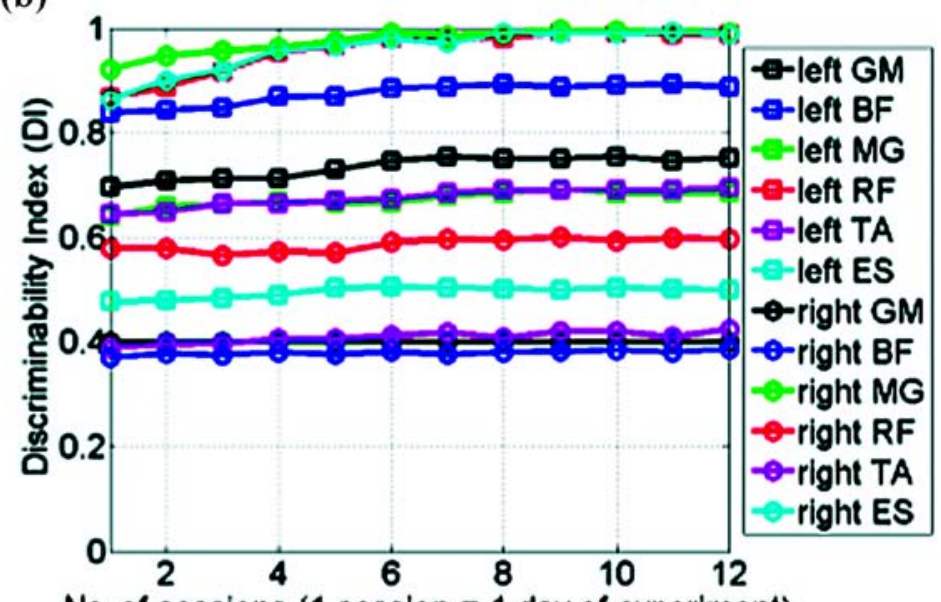

No. of sessions ( 1 session $=1$ day of experiment)

(d)

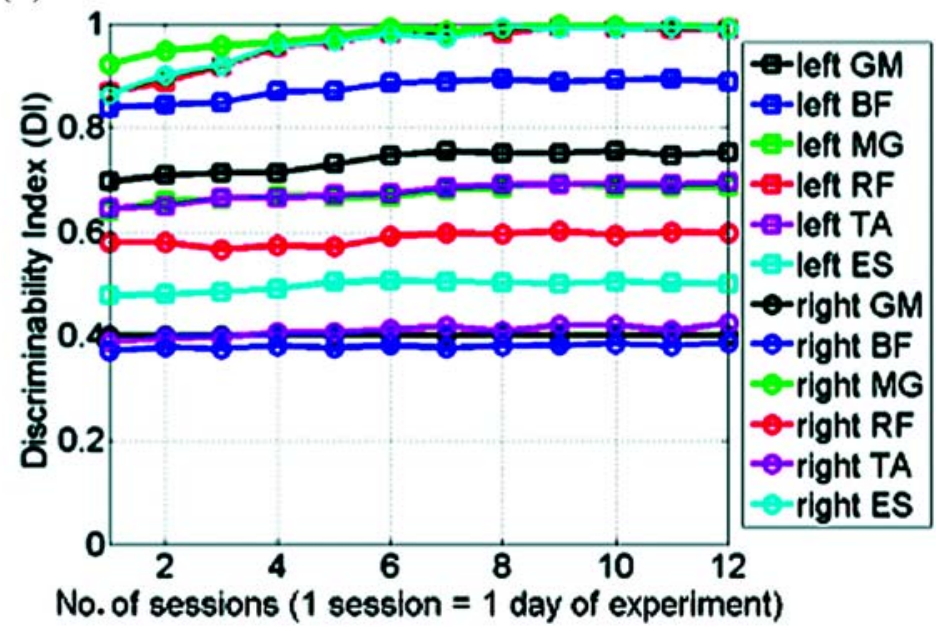

Figure 6.

Session-to-session changes in DI during month-long data collection for participant 2 with incomplete spinal cord injury (total 12 sessions): (a) left-step threshold-based classifier (TC), (b) left-step pattern recognition classifier (PRC), (c) right-step TC, and (d) right-step PRC. (For leftstep classifiers, left side is ipsilateral and right side is contralateral. For right-step classifiers, right side is ipsilateral and left side is contralateral.) $\mathrm{BF}=$ biceps femoris, $\mathrm{ES}=$ erector spinae, $\mathrm{GM}=$ gluteus medius, $\mathrm{MG}=$ medial gastrocnemius, $\mathrm{RF}=$ rectus femoris, $\mathrm{SD}=$ standard deviation, $\mathrm{TA}=$ tibialis anterior.

by the left MG (mean DI $=0.87$ ), and the contralateral right $\mathrm{RF}$ (mean DI $=0.85$ ) (for left-step classifier, right side is contralateral) was the best for identifying the intent to take a left step.

As for the nondisabled participants and participant iSCI-1, the PRC (mean DI $\mathrm{PRC}=0.72$ ) consistently outperformed the TC (mean $\mathrm{DI}_{\mathrm{TC}}=0.46$ ) for the left step in iSCI-2. For the right step, the right MG (mean DI $=0.88$ ) was the best command source followed by the right ES (mean DI $=0.85$ ) and the contralateral left RF (mean
$\mathrm{DI}=0.85$ ), and the PRC (mean $\mathrm{DI}_{\mathrm{PRC}}=0.69$ ) slightly outperformed the TC (mean $\mathrm{DI}_{\mathrm{TC}}=0.55$ ) for this unilaterally involved participant.

\section{DISCUSSION}

Ipsilateral ES and MG consistently performed well as signal sources in detecting the intent to initiate a step in both nondisabled and participants with iSCI, regardless 
Table 2.

Mean \pm SD values of DI of left and right-step classifiers for participant 1 with incomplete spinal cord injury by muscle and classifier type. Muscles tested were GM, BF, MG, RF, TA, and ES (at 9th thoracic vertebra) and classifiers were PRC and TC. SD of DI was over 10 random partitions (i.e., 10-fold cross validation).

\begin{tabular}{|c|c|c|c|c|}
\hline \multirow{2}{*}{ Muscle } & \multicolumn{2}{|c|}{ Left-Step Classifiers } & \multicolumn{2}{|c|}{ Right-Step Classifiers } \\
\hline & $\mathrm{DI}_{\mathrm{PRC}} \pm \mathrm{SD}\left(\mathrm{DI}_{\mathrm{PRC}}\right)$ & $\mathrm{DI}_{\mathrm{TC}} \pm \mathrm{SD}\left(\mathrm{DI}_{\mathrm{TC}}\right)$ & DI $_{\text {PRC }} \pm$ SD (DI PRC $)$ & $\mathrm{DI}_{\mathrm{TC}} \pm \mathrm{SD}\left(\mathrm{DI}_{\mathrm{TC}}\right)$ \\
\hline$\overline{\text { Left GM }}$ & $0.77 \pm 0.06$ & $0.66 \pm 0.06$ & $0.48 \pm 0.07$ & $0.57 \pm 0.06$ \\
\hline Left MG & $0.81 \pm 0.02$ & $0.77 \pm 0.06$ & $0.68 \pm 0.06$ & $0.59 \pm 0.04$ \\
\hline Left RF & $0.72 \pm 0.04$ & $0.57 \pm 0.05$ & $0.66 \pm 0.05$ & $0.55 \pm 0.05$ \\
\hline Left TA & $0.63 \pm 0.08$ & $0.58 \pm 0.06$ & $0.53 \pm 0.05$ & $0.56 \pm 0.07$ \\
\hline Right GM & $0.50 \pm 0.04$ & $0.53 \pm 0.03$ & $0.77 \pm 0.04$ & $0.53 \pm 0.03$ \\
\hline Right BF & $0.56 \pm 0.04$ & $0.56 \pm 0.05$ & $0.52 \pm 0.04$ & $0.52 \pm 0.06$ \\
\hline Right MG & $0.58 \pm 0.04$ & $0.56 \pm 0.06$ & $0.85 \pm 0.05$ & $0.75 \pm 0.05$ \\
\hline Right RF & $0.65 \pm 0.08$ & $0.53 \pm 0.06$ & $0.69 \pm 0.05$ & $0.53 \pm 0.05$ \\
\hline Right TA & $0.56 \pm 0.05$ & $0.55 \pm 0.06$ & $0.68 \pm 0.04$ & $0.56 \pm 0.05$ \\
\hline
\end{tabular}

$\overline{\mathrm{BF}}$ = biceps femoris, $\mathrm{DI}=$ Discriminability Index, ES = erector spinae, $\mathrm{GM}=$ gluteus medius, $\mathrm{MG}=$ medial gastrocnemius, $\mathrm{PRC}=$ pattern recognition classifier, $\mathrm{RF}=$ rectus femoris, $\mathrm{SD}=$ standard deviation, $\mathrm{TA}=$ tibialis anterior, $\mathrm{TC}=$ threshold-based classifier.

of side. These muscles were not targeted for electrical stimulation in the participants with iSCI and were able to yield clean signals unaffected by stimulation artifact. We selected the two best muscles for the command source and the type of classifier with post hoc analysis using the critical values found from Scheffé $S$ procedure after twoway ANOVA of the DI. Left ES and right ES were the best command sources for participant iSCI-1, who exhibited a symmetrical paralysis and was susceptible to muscle spasms in his MG, which decreased its reliability as a command source. In contrast, for iSCI-2, the left ES and right MG were the best command sources for the left and right steps, respectively. This participant was more impaired on his left side, which exhibited a similar result as participant iSCI-1. However, his right leg was less susceptible to spasms, which allowed the MG to be used as a robust and reliable command source as in the nondisabled cases.

The PRC consistently performed better than the TC in both nondisabled and participants with iSCI. The basic PRC presented in this article used an ensemble average of the LEs in the True class as the feature for pattern recognition, and its performance may be improved even further with better feature extraction techniques, such as principal component analysis [5].

The DI of the leading candidates for muscle command sources improved for both participants with iSCI during the month-long data collection. The DI in the case of the bilaterally involved participant iSCI-1 took longer to reach steady state as compared with unilaterally involved participant iSCI-2. The participants with iSCI were asked to use their redundant motor function during their switchtriggered FES-assisted walking. The improvement in the DI of the PRC showed that the participants with iSCI were relearning consistent volitional EMG patterns that were required to trigger the PRC of the corresponding step during FES-assisted walking.

This observation illustrates a potential benefit of EMG control over switch-triggering or other method of progressing through the stimulation patterns for walking. Incorporating the volitional activity of the partially paralyzed muscles that were previously involved in the motor activity of walking may have therapeutic effects because of the exercise and practice required to reincorporate them into functional walking patterns. On the contrary, reliance on switch triggering or automatic cycling through the stimulation patterns may reinforce the nonuse and further atrophy of the affected musculature. In addition, EMG control may give users options to naturally modulate walking speed based on the activity of the muscles selected for command sources without the need to select preprogrammed stimulus patterns for various walking velocities. Such systems may facilitate maneuvering in otherwise inaccessible environments, negotiating architectural barriers and avoiding 
(a)

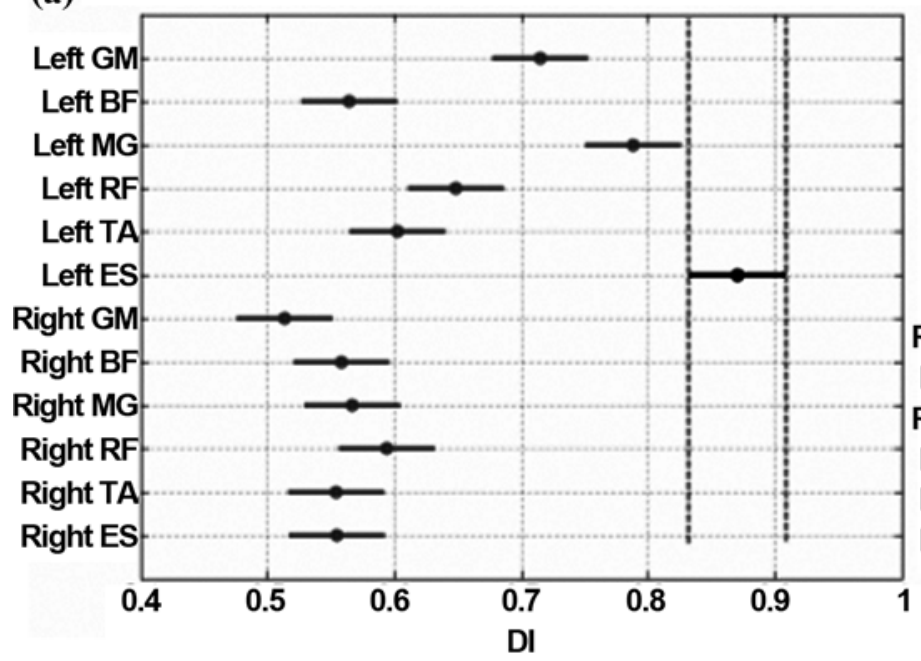

(c)

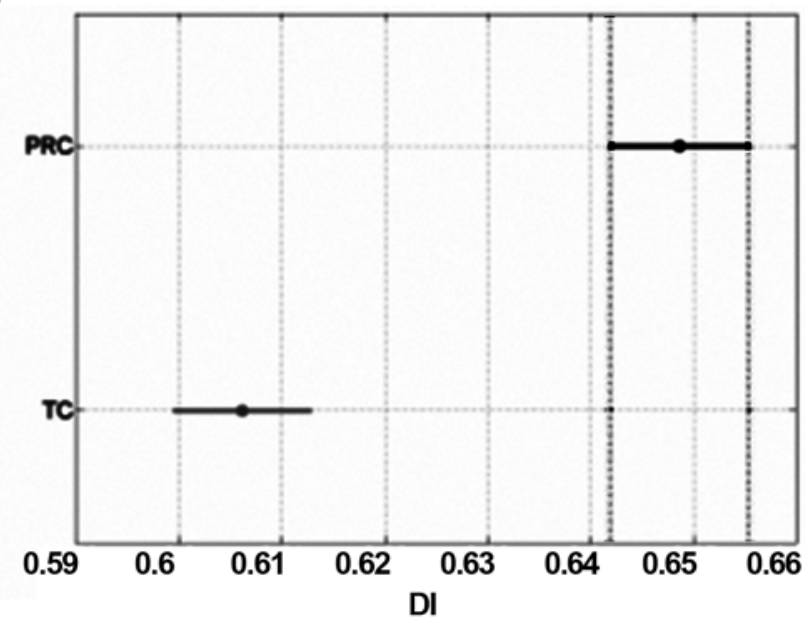

(b)

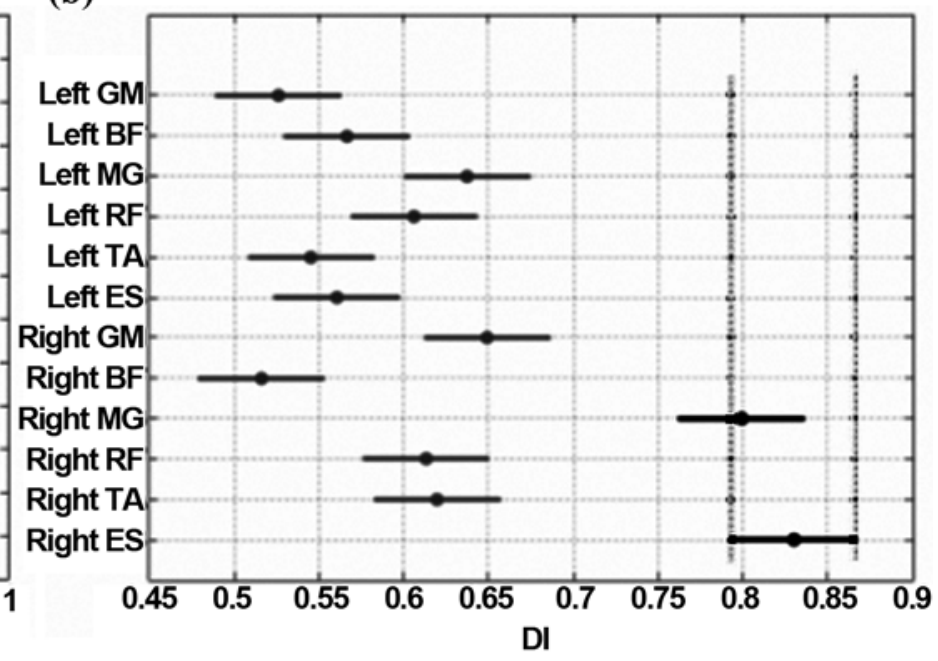

(d)

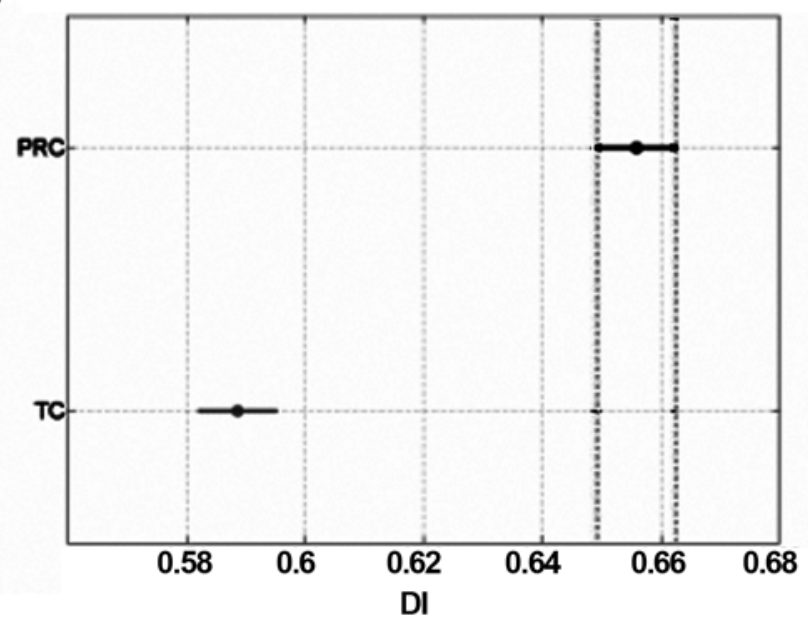

Figure 7.

Post hoc analysis of Discriminability Index (DI) with critical values $(\alpha=0.05)$ from Scheffé $S$ procedure by (a) and (b) muscle and (c) and (d) classifier type for participant 1 with incomplete spinal cord injury. (a) and (c) are for left-step classifier, while (b) and (d) are for right-step classifier. Muscles tested are gluteus medius (GM), biceps femoris (BF), medial gastrocnemius (MG), rectus femoris (RF), tibialis anterior (TA), and erector spinae (ES) (at 9th thoracic vertebra). Binary classifiers tested are pattern recognition classifier (PRC) and threshold-based classifier (TC). True mean with 95\% confidence interval is shown for highlighted (a) and (b) muscle and (c) and (d) classifier and others in pairwise comparison. Mean values are said to be significantly different if their intervals do not overlap.

obstacles, or traveling over uneven surfaces. Furthermore, individuals with iSCI exhibiting upper-limb impairments (incomplete tetraplegia is the largest subgroup of the SCI population) who may have difficulty manipulating controls of switch-triggered devices may benefit from such myoelectric systems that do not rely extensively on manual dexterity.

This feasibility study has demonstrated a method to select command sources accurately and reliably based on surface EMG from partially paralyzed muscles for myoelectrically controlled neuroprostheses employing FES to restore or enhance ambulation after incomplete SCI. Such myoelectrically controlled FES has been successfully implemented in upper-limb neuroprostheses [26]. Moreover, the objective method presented in this article is applicable to intramuscular EMG as well, e.g., one can first select the best muscle set from surface EMG and then select the best location in those muscles from fine-wire 
Table 3.

Mean \pm SD values of DI of left and right-step classifiers for participant 2 with incomplete spinal cord injury by muscle and classifier type. Muscles tested were GM, BF, MG, RF, TA, and ES (at 9th thoracic vertebra) and classifiers were PRC and TC. SD of DI was over 10 random partitions (i.e., 10-fold cross validation).

\begin{tabular}{|c|c|c|c|c|}
\hline \multirow{2}{*}{ Muscle } & \multicolumn{2}{|c|}{ Left-Step Classifiers } & \multicolumn{2}{|c|}{ Right-Step Classifiers } \\
\hline & DI $_{P_{R C}} \pm$ SD (DI PRC $)$ & $\mathrm{DI}_{\mathrm{TC}} \pm \mathrm{SD}\left(\mathrm{DI}_{\mathrm{TC}}\right)$ & $\mathrm{DI}_{\mathrm{PRC}} \pm \mathrm{SD}\left(\mathrm{DI} \mathrm{PRC}_{\mathrm{PRC}}\right)$ & $\mathrm{DI}_{\mathrm{TC}} \pm \mathrm{SD}\left(\mathrm{DI}_{\mathrm{TC}}\right)$ \\
\hline$\overline{\text { Left GM }}$ & $0.49 \pm 0.07$ & $0.00 \pm 0.00$ & $0.74 \pm 0.00$ & $0.69 \pm 0.02$ \\
\hline Left BF & $0.47 \pm 0.03$ & $0.00 \pm 0.00$ & $0.88 \pm 0.01$ & $0.59 \pm 0.03$ \\
\hline Left MG & $0.99 \pm 0.00$ & $0.74 \pm 0.06$ & $0.68 \pm 0.01$ & $0.66 \pm 0.02$ \\
\hline Left RF & $0.46 \pm 0.04$ & $0.68 \pm 0.03$ & $0.99 \pm 0.00$ & $0.69 \pm 0.02$ \\
\hline Left TA & $0.47 \pm 0.00$ & $0.00 \pm 0.00$ & $0.68 \pm 0.02$ & $0.70 \pm 0.02$ \\
\hline Left ES & $0.99 \pm 0.00$ & $0.87 \pm 0.03$ & $0.49 \pm 0.01$ & $0.57 \pm 0.02$ \\
\hline Right GM & $0.83 \pm 0.00$ & $0.71 \pm 0.03$ & $0.40 \pm 0.02$ & $0.00 \pm 0.00$ \\
\hline Right BF & $0.87 \pm 0.00$ & $0.60 \pm 0.02$ & $0.37 \pm 0.02$ & $0.00 \pm 0.00$ \\
\hline Right MG & $0.83 \pm 0.01$ & $0.69 \pm 0.03$ & $0.99 \pm 0.00$ & $0.75 \pm 0.06$ \\
\hline Right RF & $0.99 \pm 0.00$ & $0.71 \pm 0.02$ & $0.59 \pm 0.01$ & $0.56 \pm 0.04$ \\
\hline Right TA & $0.82 \pm 0.01$ & $0.48 \pm 0.02$ & $0.41 \pm 0.02$ & $0.58 \pm 0.02$ \\
\hline Right ES & $0.41 \pm 0.08$ & $0.00 \pm 0.00$ & $0.99 \pm 0.00$ & $0.71 \pm 0.05$ \\
\hline
\end{tabular}

$\overline{\mathrm{BF}}=$ biceps femoris, $\mathrm{DI}=$ Discriminability Index, $\mathrm{ES}=$ erector spinae, $\mathrm{GM}=$ gluteus medius, $\mathrm{MG}=$ medial gastrocnemius, $\mathrm{PRC}=$ pattern recognition classifier, $\mathrm{RF}=$ rectus femoris, $\mathrm{SD}=$ standard deviation, $\mathrm{TA}=$ tibialis anterior, $\mathrm{TC}=$ threshold-based classifier.

EMG for implanting of intramuscular electrodes. Intramuscular electrodes were shown to be more selective and sensitive than surface electrodes in detecting EMG signals from adjacent muscles [27] and may improve the classification accuracy. We envision future myoelectrically controlled neuroprostheses incorporating the command inputs determined by such methods to operate as described in Dutta et al. $[5,28]$. To proceed to EMG-triggered walking, users will have to trigger the FES-assisted sit-to-stand transition as well as the first FES-assisted stepping with a manual switch and thereafter trigger the subsequent steps using EMG based on TC or PRC. Users will be able to modulate the gait speed as well as stop at any time using the volitional EMG. After stopping, they will have to trigger the FES-assisted stand-to-sit transition with a manual switch. Research has shown that such EMG-triggered FES produces better coordination during stand-to-walk transition than either switch-triggered or autotriggered (electrical stimulation patterns cycled at a fixed rate) FES [29].

\section{CONCLUSIONS}

This study demonstrated that the DI is a robust metric for evaluating partially paralyzed muscles and selecting command sources objectively for myoelectrically controlled gait-assist neuroprostheses based on surface EMG with post hoc procedures. However, the best muscle set to extract a command signal may vary from person to person, depending on the individual extent and severity of paralysis. Bilateral ES was the best command source for participant iSCI-1 because of the symmetrical nature of his paralysis and plantar flexion spasms, while the left ES and right MG were the best command sources for iSCI-2 who presented with more involved left-side paralysis. In spite of intersubject variability, ipsilateral GM and ES consistently performed well in discriminating between the intent to step and all other activity in nondisabled participants as well as participants with iSCI. The participants with iSCI learned to generate consistent EMG patterns during this study, which improved the performance of the PRC. Moreover, during offline analysis, the PRC performed better than the TC in terms of DI.

However, considering the heterogeneous nature of injury and recovery after iSCI, we find that drawing a broad and generalizable conclusion is difficult. This article presented a quantitative technique for selecting EMG command sources from partially paralyzed muscles in presence of intersubject variability in the iSCI population. The participants with iSCI should be provided with enough FES-assisted gait training so that they can relearn volitional EMG patterns. During this training period, the binary classifier (TC and PRC) performance can be monitored in terms of their DI. After a steady state is reached, one can use the post hoc procedures described in this 


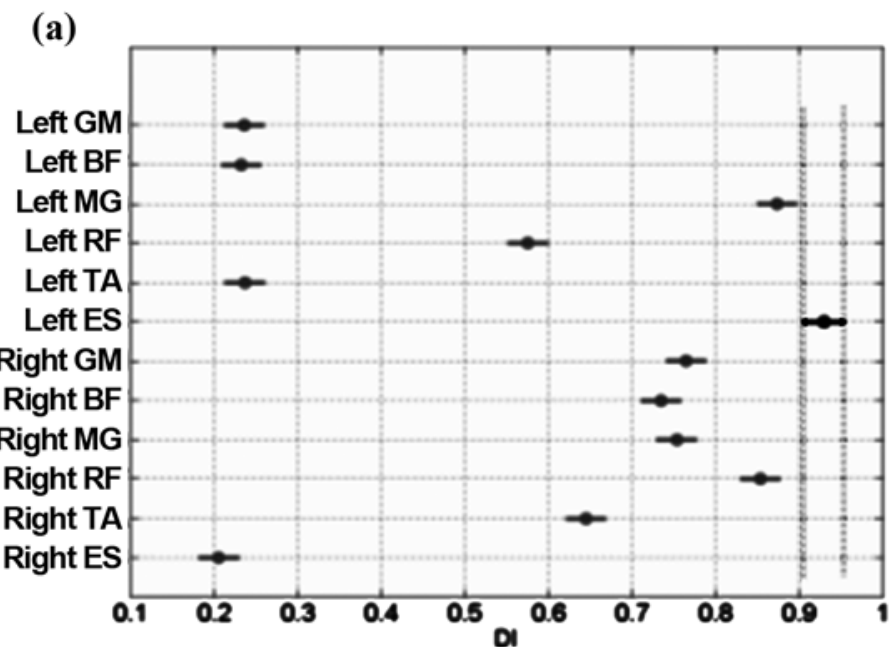

(c)

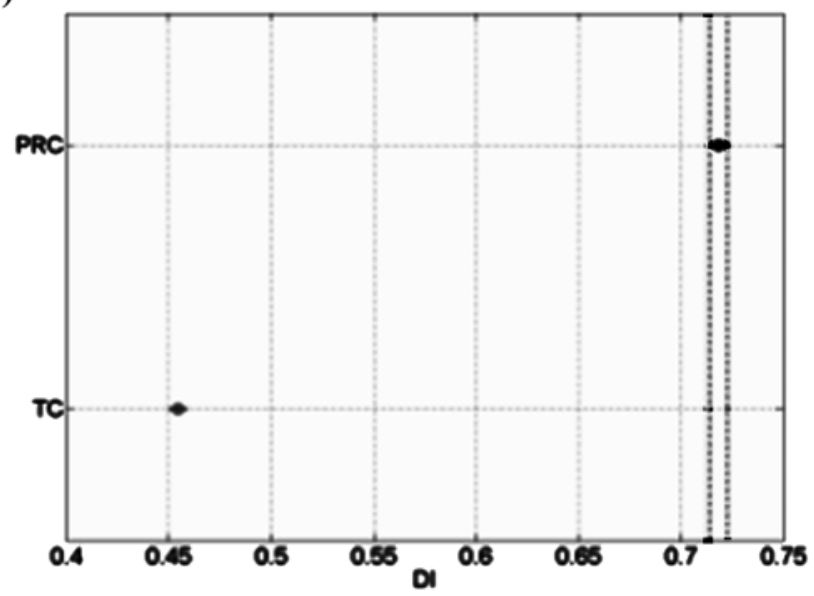

(b)

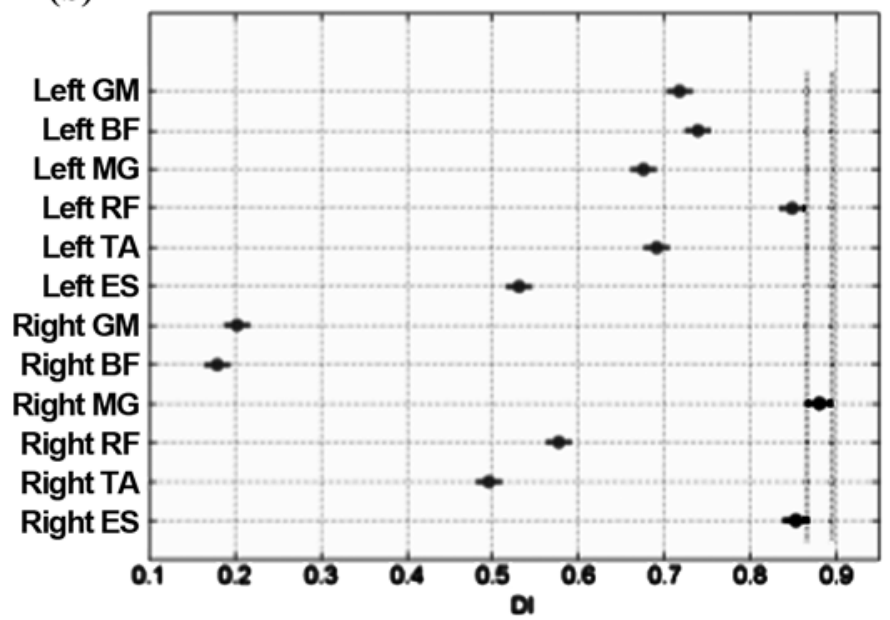

(d)

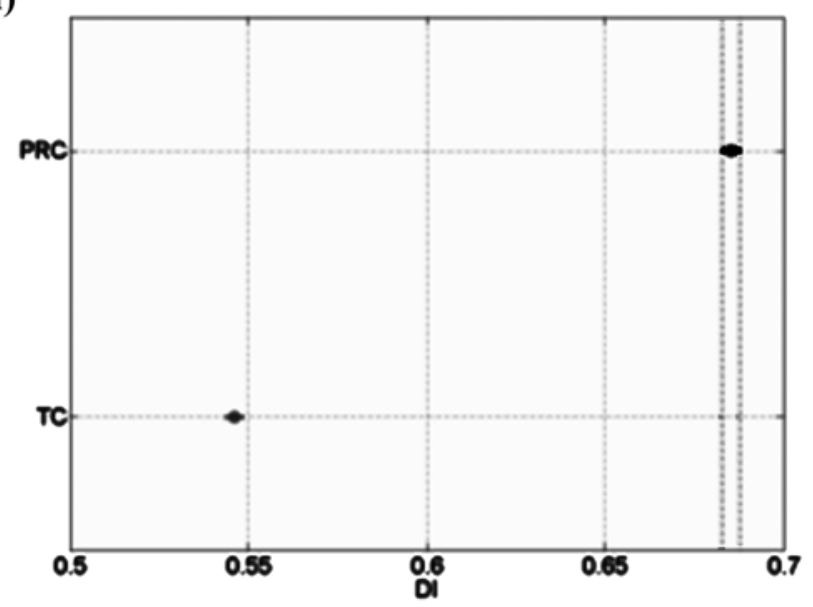

Figure 8.

Post hoc analysis of Discriminability Index (DI) with critical values ( $\alpha=0.05)$ from Scheffé $S$ procedure by (a) and (b) muscle and (c) and (d) classifier type for participant 2 with incomplete spinal cord injury. (a) and (c) are for left-step classifier, while (b) and (d) are for right-step classifier. Muscles tested are gluteus medius (GM), biceps femoris (BF), medial gastrocnemius (MG), rectus femoris (RF), tibialis anterior (TA), and erector spinae (ES) (at 9th thoracic vertebra). Binary classifiers tested are pattern recognition classifier (PRC) and threshold-based classifier (TC). True mean with 95\% confidence interval is shown for highlighted (a) and (b) muscle and (c) and (d) classifier and others in pairwise comparison. Mean values are said to be significantly different if their intervals do not overlap.

study to identify two best EMG command sources for triggering FES-assisted gait, which is a valuable tool for testing the feasibility and optimizing system performance before installation of an implanted stimulator-telemeter using implanted EMG electrodes.

\section{ACKNOWLEDGMENTS}

\section{Author Contributions:}

Study concept and design: A. Dutta, R. Kobetic, R. J. Triolo. Acquisition of data: A. Dutta.
Analysis and interpretation of data: A. Dutta, R. Kobetic, R. J. Triolo. Drafting of manuscript: A. Dutta, R. Kobetic, R. J. Triolo.

Critical revision of manuscript for important intellectual content:

A. Dutta, R. Kobetic, R. J. Triolo.

Statistical analysis: A. Dutta.

Obtained funding: R. J. Triolo.

Administrative, technical, or material support: R. Kobetic, R. J. Triolo. Study supervision: R. J. Triolo.

Financial Disclosures: The authors have declared that no competing interests exist.

Funding/Support: This material was based on work supported by the merit review project B2933R from the Department of Veterans Affairs Rehabilitation Research and Development Service. 
DUTTA et al. An objective method for selecting myoelectric command sources

Additional Contributions: We gratefully acknowledge the help from the staff at the Motion Study Laboratory of the Louis Stokes Cleveland Department of Veterans Affairs Medical Center. Dr. Anirban Dutta is now with the Neural Engineering Center for Artificial Limbs at the Rehabilitation Institute of Chicago.

Institutional Review: We obtained informed consent from all participants before their participation and all study-related procedures were approved by the institutional review board of the Louis Stokes Cleveland Department of Veterans Affairs Medical Center.

Participant Follow-Up: The authors do not plan to inform participants of the publication of this study. However, participants have been encouraged to check the study Web site for updated publications.

\section{REFERENCES}

1. Graupe D, Kohn KH. Functional electrical stimulation for ambulation by paraplegics. Malabar (FL): Krieger; 1994.

2. Kralj A, Bajd T. Functional electrical stimulation: Standing and walking after spinal cord injury. Boca Raton (FL): CRC Press; 1989.

3. Kobetic R, Triolo RJ, Uhlir JP, Bieri C, Wibowo M, Polando G, Marsolais EB, Davis JA Jr, Ferguson KA. Implanted functional electrical stimulation system for mobility in paraplegia: A follow-up case report. IEEE Trans Rehabil Eng. 1999;7(4):390-98. [PMID: 10609626] DOI:10.1109/86.808942

4. Smith B, Tang Z, Johnson MW, Pourmehdi S, Gazdik MM, Buckett JR, Peckham PH. An externally powered, multichannel, implantable stimulator-telemeter for control of paralyzed muscle. IEEE Trans Biomed Eng. 1998;45(4): 463-75. [PMID: 9556963]

DOI:10.1109/10.664202

5. Dutta A, Kobetic R, Triolo RJ. Ambulation after incomplete spinal cord injury with EMG-triggered functional electrical stimulation. IEEE Trans Biomed Eng. 2008;55(2 Pt 1):791-94. [PMID: 18270018] DOI:10.1109/TBME.2007.902225

6. Bhadra N, Kilgore KL, Peckham PH. Implanted stimulators for restoration of function in spinal cored injury. Med Eng Phys. 2001;23(1):19-28. [PMID: 11344004] DOI:10.1016/S1350-4533(01)00012-1

7. Pappas IP, Popovic MR, Keller T, Dietz V, Morari M. A reliable gait phase detection system. IEEE Trans Neural Syst Rehabil Eng. 2001;9(2):113-25. [PMID: 11474964] DOI:10.1109/7333.928571

8. Mansfield A, Lyons GM. The use of accelerometry to detect heel contact events for use as a sensor in FES assisted walking. Med Eng Phys. 2003;25(10):879-85.

[PMID: 14630475]

DOI:10.1016/S1350-4533(03)00116-4

9. Sinkjaer T, Haugland M, Inmann A, Hansen M, Nielsen KD. Biopotentials as command and feedback signals in functional electrical stimulation systems. Med End Phys. 2003;25(1):29-40. [PMID: 12485784$]$ DOI:10.1016/S1350-4533(02)00178-9

10. Lauer RT, Smith BT, Betz RR. Application of a neurofuzzy network for gait event detection using electromyography in the child with cerebral palsy. IEEE Trans Biomed Eng. 2005;52(9):1532-40. [PMID: 16189966]

DOI:10.1109/TBME.2005.851527

11. Graupe D, Kordylewski H. Artificial neural network control of FES in paraplegics for patient responsive ambulation. IEEE Trans Biomed Eng. 1995;42(7):699-707.

[PMID: 7622153] DOI:10.1109/10.391169

12. Cavanagh PR, Komi PV. Electromechanical delay in human skeletal muscle under concentric and eccentric contractions. Eur J Appl Physiol Occup Physiol. 1979;42(3): 159-63. [PMID: 527577] DOI:10.1007/BF00431022

13. Kordylewski H, Graupe D. Control of neuromuscular stimulation for ambulation by complete paraplegics via artificial neural networks. Neurol Res. 2001;23(5):472-81.

[PMID: 11474803] DOI:10.1179/016164101101198866

14. Thorsen R, Spadone R, Ferrarin M. A pilot study of myoelectrically controlled FES of upper extremity. IEEE Trans Neural Syst Rehabil Eng. 2001;9(2):161-68. [PMID: 11474969] DOI:10.1109/7333.928576

15. Futami KS, Kawanishi T, Sugiyama T, Cikajlo I, Handa Y. Application of local EMG-driven FES to incompletely paralyzed lower extremities. Proceedings of the 10th Annual Conference of the International FES Society; 2005 Jul; Montreal, Canada. p. 204-6.

16. Hardin E, Kobetic R, Murray L, Corado-Ahmed M, Pinault G, Sakai J, Bailey SN, Ho C, Triolo RJ. Walking after incomplete spinal cord injury using an implanted FES system: A case report. J Rehabil Res Dev. 2007;44(3):333-46. [PMID: 18247230]

DOI:10.1682/JRRD.2007.03.0333

17. Bailey SN, Hardin EC, Kobetic R, Boggs LM, Pinault G, Triolo RJ. Neurotherapeutic and neuroprosthetic effects of implanted functional electrical stimulation for ambulation after incomplete spinal cord injury. J Rehabil Res Dev. 2010;47(1):7-16. [PMID: 20437323]

DOI:10.1682/JRRD.2009.03.0034

18. Kobetic R, Marsolais EB. Synthesis of paraplegic gait with multichannel functional neuromuscular stimulation. IEEE Trans Rehabil Eng.1994;2(2):66-79. DOI:10.1109/86.313148

19. Kobetic R, Triolo RJ, Marsolais EB. Muscle selection and walking performance of multichannel FES systems for ambulation in paraplegia. IEEE Trans Rehabil Eng. 1997; 5(1):23-29. [PMID: 9086382] DOI:10.1109/86.559346 
20. Hermens HJ, Freriks B, Merletti R, Stegeman D, Blok J, Rau G, Disselhorst-Klug C, Hagg G. SENIAM: European recommendations for surface electromyography: Results of the SENIAM project. Enschede (the Netherlands): Roessingh Research and Development; 1999.

21. Hines AE, Crago PE, Chapman GJ, Billian C. Stimulus artifact removal in EMG from muscles adjacent to stimulated muscles. J Neurosci Methods. 1996;64(1):55-62. [PMID: 8869484] DOI:10.1016/0165-0270(95)00099-2

22. Wickens TD. Elementary signal detection theory. New York (NY): Oxford University Press; 2002.

23. Bradley AP. The use of the area under the ROC curve in the evaluation of machine learning algorithms. Pattern Recognit. 1997;30(7):1145-59. DOI:10.1016/S0031-3203(96)00142-2

24. Walpole RE, Myers RH. Probability and statistic for engineers and scientists. New York (NY): Macmillan; 1990.

25. Hanley JA, McNeil BJ. The meaning and use of the area under a receiver operating characteristic (ROC) curve. Radiology. 1982;143(1):29-36. [PMID: 7063747]

26. Giuffrida JP, Crago PE. Reciprocal EMG control of elbow extension by FES. IEEE Trans Neural Syst Rehabil Eng. 2001;9(4):338-45. [PMID: 12018646] DOI:10.1109/7333.1000113

27. Chae J, Knutson J, Hart R, Fang ZP. Selectivity and sensitivity of intramuscular and transcutaneous electromyography electrodes. Am J Phys Med Rehabil. 2001;80(5):374-79. [PMID: 11327560]

DOI:10.1097/00002060-200105000-00010
28. Dutta A, Kobetic R, Triolo R. Development of an implanted intramuscular EMG-triggered FES system for ambulation after incomplete spinal cord injury. Conf Proc IEEE Eng Med Biol Soc. 2009;2009:6793-97. [PMID: 19964711]

29. Dutta A, Kobetic R, Triolo RJ. Gait initiation with electromyographically triggered electrical stimulation in people with partial paralysis. J Biomech Eng. 2009;131(8):081002. [PMID: 19604014$]$ DOI:10.1115/1.3086356

Submitted for publication August 2, 2010. Accepted in revised form February 23, 2011.

This article and any supplementary material should be cited as follows:

Dutta A, Kobetic R, Triolo RJ. An objective method for selecting command sources for myoelectrically triggered lower-limb neuroprostheses. J Rehabil Res Dev. 2011; 48(8):935-48.

DOI:10.1682/JRRD.2010.08.0141

ResearcherID: Anirban Dutta, PhD: E-5873-2011

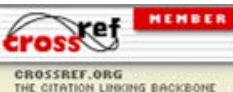

